Rev. Elev. Méd. vét. Pays trop., 1974, 27 (4) : 385-395

\title{
Un vaccin mixte trivalent contre la peste bovine, la péripneumonie et le charbon bactéridien
}

\author{
par A. PROVOST $(*)$, C. BORREDON $(*)$ et P. BOCQUET $(*)$ \\ (avec la collaboration technique de MM. N'GALDAM, N. GOMBOT, T. KAMPETE)
}

\begin{abstract}
RESUME
Après avoir indiqué les raisons qui militent en faveur de l'utilisation d'un vaccin mixte antibovipestique, antipéripneumonique et anticharbonneux en Afrique centrale, les auteurs décrivent la conception d'un tel vaccin et la méthodologie de sa préparation. Les contrôles de production et les essais sur le terrain sont commentés: la solution molaire de sulfate de magnésium, utilisée au Tchad pour la remise en solution du vaccin mixte antipestique-antipéripneumonique utilisé dans ce pays, est nocive pour les spores de $B$. anthracis.
\end{abstract}

\section{INTRODUCTION}

Il ne paraît pas nécessaire de devoir présenter des arguments militant en faveur de l'utilisation des vaccins mixtes pour la prophylaxie des grandes épizooties tropicales; ils ne peuvent que simplifier le travail et accroître l'efficacité des opérations. Encore faut-il que les associations proposées aient une réelle indication prophylactique et que l'on soit assuré que leurs différents composants n̈interfèrent pas l'un sur l'autre dans le sens d'une diminution de l'immunogénèse, comme ce fut le cas pour l'association vaccin capripestique-vaccin antipéripneumonique $(26,16)$. C'est suivant cette ligne de conduite que, depuis 1965 , le laboratoire de Farcha produit un vaccin mixte antibovipestique-antipéripneumonique (31). Initialement préparé avec la souche bovipestique de PLOWRIGHT et FERRIS (23), adaptée puis atténuée

(*) Laboratoire de Farcha, B.P. 433, N'Djamena, République du Tchad. en cultures cellulaires de rein de veau, et avec la souche $\mathrm{KH}_{3} \mathrm{~J}$ de Mycoplasma mycoides (8), rendue streptomycinorésistante par adaptation à des quantités croissantes de streptomycine (28), on a maintenant substitué à la souche $\mathrm{KH}_{3} \mathrm{~J}$ la souche $\mathrm{T}_{1}-\mathrm{SR}$ streptomycinorésistante aux qualités immunigènes de beaucoup supérieures $(4,5,12)$.

La présente note relate les circonstances ayant amené à l'introduction de l'antigène charbon bactéridien dans le vaccin mixte et les résultats que l'on a obtenus avec cette association vaccinale.

La lutte contre la peste bovine sur le continent africain (P.C. 15 de l'O.U.A./C.S.T.R.) a pendant plusieurs années mobilisé l'activité des services vétérinaires (14); de ce fait, la lutte contre les autres maladies contagieuses a été en certaines circonstances plus ou moins négligée. Ce fut le cas du Tchad pour le charbon bactéridien où la vaccination n'a plus été pratiquée dans les foyers traditionnels, comnue en témoigne le relevé suivant : 


\begin{tabular}{|c|c|c|}
\hline Années & $\begin{array}{c}\mathrm{N}^{\text {bre }} \text { de doses } \\
\text { de vaccin } \\
\text { anticharbonneux } \\
\text { délivrées }\end{array}$ & $\begin{array}{c}\mathrm{N}^{\text {bre }} \text { de doses } \\
\text { de vaccin } \\
\text { utilisées } \\
\text { (bovins) }\end{array}$ \\
\hline 1958 & 355750 & 227309 \\
\hline 1959 & 268300 & 150736 \\
\hline 1960 & 337300 & 215846 \\
\hline 1961 & 515600 & 260117 \\
\hline 1962 & 330200 & 125970 \\
\hline 1963 & 192700 & 90418 \\
\hline 1964 & 249800 & 56328 \\
\hline 1965 & 200500 & 108943 \\
\hline 1966 & 107000 & 79369 \\
\hline \multicolumn{3}{|c|}{$\begin{array}{c}\text { (dont } 15000 \text { dans le nord-ouest, } \\
0 \text { dans le centre-ouest) }\end{array}$} \\
\hline \multicolumn{3}{|c|}{$\begin{array}{c}\text { (dont } 17143 \text { dans le nord-ouest, } \\
0 \text { dans le centre-ouest) }\end{array}$} \\
\hline $\begin{array}{l}1969 \\
\text { (dont }\end{array}$ & $\begin{array}{c}75500 \\
\text { dans le nord-oues }\end{array}$ & $\begin{array}{c}27559 \\
\text { et le centre-ou }\end{array}$ \\
\hline
\end{tabular}

Les années 1970 et 1971 ont vu en Afrique centrale une recrudescence de l'enzootie bactéridienne, en partie due à des conditions écologiques spéciales (abondance des eaux de surface après plusieurs années de sécheresse, augmentation importante du trafic de bétail de commerce dans l'ouest tchadien) mais aussi à une désaffection progressive de la vaccination dans les terroirs infectés jouxtant le lac Tchad pour la raison déjà invoquée et aussi par manque de moyens d'action. De nombreux cas humains ont été enregistrés (35).

Il aurait été simple de préconiser le retour à la vaccination avec le vaccin anticharbonneux classique (souche Sterne $34 \mathrm{~F} 2$ en suspension glycérinée), ce qui au demeurant a été fait pour parer au plus pressé. Toutefois, l'exemple d'autres pays d'élevage extensif, dont le Turkestan et l'Afrique du sud (37) a montré que la simple vaccination, généralisée et soutenue pendant plusieurs années, amenait à l'éradication de la maladie par amenuisement des sources du contage. C'est bien là le but à atteindre en Afrique centrale, d'où l'idée de la préparation d'un vaccin mixte destiné aux bovins, incorporant l'antigène charbonneux qui serait inoculé annuellement en profitant de la vaccination antipestique obligatoire. On conçoit toutefois d'emblée que, pour arriver au but recherché dans ce plan de prophylaxie, les chameaux, petits ruminants et équidés, ne doivent pas être négligés.

\section{MATERIEL ET METHODES}

\section{Conception du vaccin mixte}

La solution logique à adopter pour arriver au but recherché était tout simplement d'incorporer aux liquides de culture du virus bovipestique et à la culture en bouillon de la souche $\mathrm{T}_{1}-\mathrm{SR}$ de $M$. mycoides une suspension de spores de la souche $34 \mathrm{~F} 2$ de Bacteridium anthracis avant de procéder aux opérations de lyophilisation. Après l'avoir envisagée, on a écarté la solution qui consistait à remplacer une partie du diluant de lyophilisation par une culture en dialyse du microbe charbonneux selon les principes de GLADSTONE (9), car la technologie de la production de cet antigène est encore loin d'être codifiée pour en faire un vaccin d'un prix de revient compétitif avec celui des vaccins sporulés (15); par ailleurs, il n'est immunigène qu'en assez grosses doses et requiert deux inoculations pour parfaire une immunité valable.

Toutefois, les cultures du virus bovipestique et de la souche $\mathbf{T}_{1}$ de $M$. mycoides nécessitent l'incorporation d'antibiotiques dans les milieux, antibiotiques dont on peut craindre a priori qu'ils ne soient délétères pour $B$. anthracis. Dans un premier temps, on a songé à répéter pour la souche Sterne le procédé de sélection de mutants qui avait été mis sur pied pour les souches $\mathrm{KH}_{3} \mathrm{~J}$ et $\mathrm{T}_{1}$ de $M$. mycoides (28): recherche de mutants insensibles à l'action de la pénicilline et à la streptomycine, antibiotiques présents dans le milieu de culture du virus bovipestique, par culture de la souche dans des milieux à concentrations croissantes de ces antibiotiques. Des essais, aux résultats négatifs, ont été entrepris en ce sens.

A la réflexion, on s'est dit que la concentration de ces deux antibiotiques dans la dose vaccinale était minime et ne devait pas être toxique pour les spores charbonneuses. En effet, les liquides de cultures cellulaires du virus bovipestique contiennent par $\mathrm{ml}$ : 100 unités de pénicilline et $100 \mu \mathrm{g}$ de streptomycine (la fungizone, présente elle aussi, est sans action sur le microbe charbonneux). Une partie de la pénicilline se dégrade au cours de l'incubation 
du virus. Par la suite, lors de la préparation du vaccin, les liquides de cultures cellulaires seront dilués au $1 / 10$ dans la culture de $M$. mycoides et le support de lyophilisation. Lors de sa reconstitution sur le terrain, le vaccin (présenté en flacons de 100 doses contenant l'équivalent de $5 \mathrm{ml}$ de liquide avant sa lyophilisation) sera dilué au $1 / 20$ avec le liquide de reconstitution avant d'être injecté sous la peau de l'animal à vacciner; la dose est de $1 \mathrm{ml}$. En d'autres termes, les spores charbonneuses seront exposées à $10 \mathrm{U}$ de pénicilline et $10 \mu \mathrm{g}$ de streptomycine lors de la confection du mélange, et à $0,4 \mathrm{U}$ de pénicilline et $0,4 \mu \mathrm{g}$ de streptomycine par $\mathrm{ml}$ lors de la reconstitution du vaccin. Gardant à l'esprit que ces deux antibiotiques sont actifs sur des bactéries en voie de développement (38) il y a peu de chances pour que leur présence soit néfaste aux spores, pour autant qu'elles ne commencent pas à germer pendant les opérations de confection du vaccin. Pour arrêter cet éventuel processus, il suffit tout simplement de pratiquer ces opérations avec des liquides réfrigérés qui, de par leur température, se montreront dysgénésiques pour la germination des spores. Dans le vaccin reconstitué prêt à être employé, la concentration des antibiotiques est alors bien trop faible pour être nuisible.

\section{Confection du vaccin mixte}

a) Antigènes bovipestique et péripneumonique. -- Le mode de préparation de ces deux antigènes ne se départ que de peu de ce qui a déjà été amplement décrit (31); seule la culture de la souche $\mathrm{T}_{1}-\mathrm{SR}$ de $M$. mycoides, particulièrement luxuriante en milieu F-66, est arrêtée au bout de 48 heures au lieu de 72 heures pour la souche $\mathrm{KH}_{3} \mathrm{~J}$-SR. Le calendrier des opérations est donc décalé d'un jour par rapport à celui qui a été présenté.

b) Antigène charbonneux. - La production de l'antigène est faite selon les normes internationales de l'O.M.S. (19) inspirées de la technique de STERNE (36) avec la souche 34F2 du Laboratoire international des étalons biologiques à Weybridge (Angleterre). La souche conservée à l'état lyophilisé subit un premier passage sur gélose de Sterne; la culture en nappe est rincée en bouillon et la suspension obtenue sert immédiatement à ensemencer les boîtes de Roux de production contenant une couche de gélose de Sterne très sèche. Après
48 heures de culture, on récolte dans un minimum de diluant de lyophilisation (eau peptonée à 11 p. 100) que l'on chauffe à $56^{\circ} \mathrm{C}$ pendant 1 heure pour inactiver les corps microbiens et ne conserver que les spores. Cette solution-mère est conservée au réfrigérateur.

On effectue un titrage classique des spores sur gélose et en milieu liquide; un calcul statistique simple (MPN) (*) fournit une numération approchée.

c) Mélange des antigènes, répartition et lyophilisation. - Juste avant la confection du mélange, la solution-mère d'antigène charbonneux, préalablement titrée, est diluée, si besoin est, avec du diluant de lyophilisation glacé pour l'amener à $5 \times 10^{9}$ spores par ml. Ce chiffre tient compte des dilutions à venir lors de la confection du vaccin et de son utilisation sur le terrain, mais aussi des pertes possibles lors de la lyophilisation.

Le mélange est alors effectué sous agitation magnétique dans les proportions suivantes:

\section{Parties}

- Liquide de cultures cellulaires du virus bovipestique . . . . $\quad$. 1

- Culture en bouillon de la souche $\mathrm{T}_{1}$-SR

- Suspension de la souche $34 \mathrm{~F} 2$. 1

- Lait écrémé en poudre $(\mathrm{p} / \mathrm{v})$. 4,5

Le liquide de cultures cellulaires du virus bovipestique et la culture de la souche $T_{1}-S R$ sont réfrigérés avant le mélange. Au total, on aura amené $500.10^{9}$ spores charbonneuses par litre de mélange.

On répartit sous froid (récipient contenant le mélange maintenu en glace fondante) sous le volume de $5 \mathrm{ml}$ en flacons de $20 \mathrm{~cm}^{3}$, préalablement réfrigérés à $-25^{\circ} \mathrm{C}$ sur les étagères de l'appareil à lyophiliser. Chaque plateau de flacons remplis est aussitôt replacé dans le lyophilisateur.

La lyophilisation est conduite sur 36 heures, avec dessiccation primaire à $-25^{\circ} \mathrm{C}$, température qui garantit le succès de la lyophilisation des mycoplasmes.

Chaque flacon lyophilisé est donné pour 100 doses de vaccin de $1 \mathrm{ml}$ chacune.

(*) Méthode MPN ou most probable number. 


\section{Contrôles du vaccin mixte}

\section{A. Contrôles de titre}

Le contrôle du titre de l'antigène charbonneux s'effectue classiquement par numération en tubes de milieu de culture de dilutions décimales (MPN) du vaccin reconstitué depuis 30 minutes à la dose vaccinale (dilution de la pastille lyophilisée dans $100 \mathrm{ml}$ de sérum physiologique réfrigéré). Le minimum requis par les normes internationales est de 2 à 10 millions de spores cultivables par dose vaccinale.

Le titrage effectué assigne au vaccin mixte préparé la richesse de $7 \pm 1$ millions de spores viables par dose. Par rapport à ce qui a été introduit lors de la confection du vaccin, il $\mathrm{y}$ a une perte d'environ les $2 / 3$, où il est difficile de déceler la part de ce qui peut être dû éventuellement aux antibiotiques ou à la lyophilisation.

Les contrôles des composants pestique et péripneumonique s'effectuent selon les techniques habituelles de numération $(31,25)$ après avoir dilué le contenu du flacon de vaccin dans $100 \mathrm{ml}$ de sérum physiologique glacé contenant $1 \mathrm{mg}$ d'oxacilline et de colimycine par $\mathrm{ml}$. Le titrage de la souche $\mathrm{T}_{1}-\mathrm{SR}$ de $M$. mycoides est pratiqué en tubes de milieu F-66 contenant ces deux antibiotiques pour inhiber le développement des spores charbonneuses. Le minimum requis est de $10^{2,5}$ DCP pour le virus pestique et de $10^{7}$ germes viables pour $M$. mycoides $(20,10)$. Ces titres sont largement dépassés dans la pratique : $10^{3,2}$ pour la peste, $9.10^{8}$ pour la péripneumonie.

\section{B. Contrôles d'innocuité}

On pouvait se demander si l'adjonction de l'antigène bactéridien au vaccin mixte antipestique-antipéripneumonique n'aurait pas un effet fâcheux en favorisant au point d'inoculation sous-cutanée l'apparition d'une réaction willemsienne, analogue au phénomène que MORNET, ORUE et DIAGNE (18) ont relaté.

Un test d'innocuité est donc mis sur pied, utilisant 6 bouvillons sans anticorps antibovipestiques neutralisants ni anticorps antipéripneumoniques fixant le complément. Achetés dans un marché éloigné du nord-ouest du Tohad et dépourvus de traces auriculaires en trèfle normalement apposées par les équipes de vaccination, on peut être assuré de leur pleine réceptivité, affirmée de surcroît par les tests sérologiques. Les six bouvillons reçoivent par voie sous-cutanée $5 \mathrm{ml}$ du mélange vaccinal prélevé avant la lyophilisation, c'est-à-dire au moins 20 fois la dose vaccinale.

Sept moutons reçoivent par voie sous-cutanée une dose vaccinale; 3 témoins reçoivent un placebo de sérum physiologique.

\section{Contrôles d'efficacité}

Ils ont été menés sur bouvillons et sur moutons.

Sept bouvillons, achetés et contrôlés dans les mêmes conditions que ci-dessus, reçoivent par voie sous-cutanée rétroscapulaire une dose vaccinale de vaccin mixte (soit $7.10^{6}$ spores charbonneuses, $10^{2,7} \mathrm{DCP}_{50}$ de virus pestique et $9.10^{8}$ mycoplasmes) reconstituée en sérum physiologique.

Comme il est de connaissance classique que le bœuf ne se prête pas à l'étude expérimentale du charbon bactéridien, 6 nouveaux moutons et les 3 moutons témoins du contrôle d'innocuité sont eux aussi inoculés avec une dose vaccinale. Au total, il y aura donc eu 9 moutons d'inoculés pour ce test.

L'épreuve virulente intervient 5 mois plus tard sur les bovins vaccinés et sur 3 témoins, 15 jours après la vaccination sur les moutons vaccinés; on inocule $10^{6}$ spores de la souche pathogène TREC de $B$. anthracis par voie sous-cutanée au niveau de l'encolure.

\section{Comportement du vaccin mixte reconstitué en solution molaire de sulfate de magnésium}

Depuis 1970, le Service de l'Elevage du Tchad utilise pour la reconstitution et la dilution sur le terrain du vaccin mixte antibovipestique-antipéripneumonique la solution molaire (1 M) de sulfate de magnésium, qui est thermoprotectrice pour le virus bovipestique (27, 23) et pour les mycoplasmes (29). Cette pratique est dictée par la nécessité, les moyens de conservation sous froid faisant totalement défaut en brousse, d'où l'impératif de posséder un diluant thermoprotecteur du vaccin à défaut de sa reconstitution en eau distillée ou en sérum physiologique glacés. C'est dans cette optique, d'ailleurs, qu'a été développée une souche vaccinale du virus bovipestique à dégradation ther- 
mique retardée à la température de $45^{\circ} \mathrm{C}$ à l'état lyophile (30).

On devait se poser la question du comportement des spores charbonneuses dans un milieu sulfatomagnésien fortement hypertonique. A cet effet, on reconstitue la pastille vaccinale lyophilisée d'un flacon de vaccin mixte dans $100 \mathrm{ml}$ de solution molaire de sulfate de magnésium à température du laboratoire $\left(33^{\circ} \mathrm{C}\right)$ et l'on effectue au bout de $30 \mathrm{mn}$ à la même température des titrages des spores charbonneuses en milieu liquide.

\section{Essais sur le terrain}

Lorsque l'on a été assuré que le vaccin était inoffensif pour les bovins, un essai à assez grande échelle dans des conditions contrôlées a été monté sur le terrain.

Il est pratiqué sur 234 veaux âgés de 6 à 24 mois appartenant à des éleveurs de deux campements reculés de l'ouest du Kanem, près de la frontière du Niger. Le lieu a été choisi parce que, depuis 2 ans, les tournées de vaccination étaient négligées dans cet endroit pour des raisons diverses; on était donc assuré de trouver de jeunes bovins sans anticorps antipestiques. Par ailleurs, la péripneumonie étant inconnue dans cette région, on pouvait mesurer la réponse sérologique, au demeurant faible et inconstante, à l'antigène péripneumonique. Il n'y avait pas non plus de raison a priori pour que les animaux aient été vaccinés contre le charbon bactéridien.

Chacun d'eux reçoit une dose vaccinale par voie sous-cutanée rétroscapulaire du vaccin dilué en sérum physiologique glacé; le flacon de vaccin dilué est conservé en glace fondante pendant la durée des opérations.

Une prise de sang est effectuée à la veine jugulaire avant puis un mois après la vaccination.

Enfin, on était prêt à intervenir avec un antibiotique mycoplasmocide et bactéricide (spiramycine) $\left(^{*}\right)$ si des réactions locales fâcheuses s'étaient produites (17).

$\mathrm{Au}$ laboratoire, on recherche les anticorps antibovipestiques, antipéripneumoniques et anticharbonneux.

(*) Suanovil Spécia.
Etant donné les circonstances de l'essai, aucune épreuve virulente n'a été tentée.

\section{Techniques sénologiques}

a) Anticorps antibovipestiques. - Ils sont mesurés par séroneutralisation en cultures cellulaires de rein d'embryon de veau selon la technique de PLOWRIGHT et FERRIS (24). Le titre est exprimé en $\mathrm{TN}_{50}$ (titre neutralisant 50 p. 100) sur la base de l'exposant du $\log _{10}$.

b) Anticorps antipéripneumoniques. - On a fait appel à une technique de fixation du complément dérivée de la technique de Kolmer (33). Quoiqu'entachée de non-spécificité dans les basses dilutions des sérums examinés, elle a le mérite d'être sensible, donc de se prêter à la mesure des faibles qualités inductrices d'anticorps de la souche $T_{\mathbf{1}}-\mathrm{SR}$. Les titres sont exprimés par l'exposant du $\log _{2}$ de la dilution donnant une fixation d'au moins 75 p. 100.

c) Anticorps charbonneux. - La recherche des anticorps charbonneux n'est pas une opération classique. Dans le cas présent, elle a été choisie comme méthode d'investigation car il n'était pas possible de faire des épreuves d'immunité sur les animaux vaccinés en brousse.

La fixation du complément a été utilisée par les chercheurs américains (15) avec des résultats plus ou moins probants malgré les modifications introduites par CHO (3); les titres détectés sont faibles. Reprenant l'argumentation de BUCHANAN (1) il a paru préférable de faire appel à une technique d'hémagglutination conditionnée.

Un premier antigène est préparé en utilisant des hématies de mouton formolées, sensibilisées par une suspension en sérum physiologique formolé à 1,5 p. 100 d'une culture de 24 heures sur gélose de $B$. anthracis souche Sterne $34 \mathrm{~F} 2$, récoltée bien avant que la sporulation ne soit effective. Essayé avec différents sérums d'animaux servant aux tests d'innocuité du vaccin anticharbonneux préparé par le laboratoire, il ne s'est pas révélé satisfaisant par manque de sensibilité.

On a alors fait appel à l'antigène de PALIT et NILAKANTAN (21) consistant en une suspension d'hématies sensibilisées par un complexe polyosidique extrait d'une culture de la bactéridie. A cet effet, on récolte en sérum 
physiologique une culture de 12 heures (donc non sporulée) de la souche $34 \mathrm{~F} 2$ sur gélose à 3 p. 100 d'extrait de levure. Le complexe polyosidique est extrait selon la technique de Westphal du phénol à chaud, en mettant en œuvre la méthodologie que BUTTERY et PLACKETT (2) ont utilisée pour la galactane péripneumonique. A la concentration de $0,1 \mathrm{mg} / \mathrm{ml}$, il sert à sensibiliser une suspension à 0,25 p. 100 d'hématies de chèvre formolées. La chèvre a été choisie comme une espèce donatrice d'hématies car les sérums bovins ne contiennent pas d'hétéro-agglutinines dirigées contre elles (7). Après plusieurs lavages destinés à éliminer l'excès de polyoside, les hématies sensibilisées sont remises en suspension à 0,25 p. 100 dans un tampon phosphaté à $\mathrm{pH}: 7$ contenant 0,1 p. 100 de gélatine et 0,5 p. 100 d'albumine bovine comme stabilisateurs.

Le test est très simplement effectué par examen de dilutions en progression géométrique de raison 2 des sérums : $0,4 \mathrm{ml}$ de chaque dilution sont mélangés à $0,4 \mathrm{ml}$ de la suspension d'hématies; la lecture intervient après un séjour de 2 heures à la température du laboratoire. Des témoins sont inclus pour apprécier la stabilité de l'antigène et l'absence d'hétéroagglutinines dans les sérums. Les titres sont exprimés par l'exposant du $\log _{2}$ de la dilution du sérum donnant une hémagglutination d'au moins 75 p. 100.

\section{RESULTATS}

\section{Contrôle des titres}

Les chiffres déjà cités montrent que le vaccin mixte correspond aux normes requises pour chacun des 3 composants.

\section{Contrôles d'innocuité}

Aucun des 6 bouvillons inoculés avec $5 \mathrm{ml}$ du mélange avant lyophilisation n'a présenté d'hyperthermie ni de réaction locale.

Par contre, les 7 moutons inoculés avec une dose vaccinale sont morts entre le $12^{\mathrm{e}}$ et le $25^{\mathrm{e}}$ jour après l'injection. Rien à l'autopsie ne permettait de penser à l'infection charbonneuse. Cowdria ruminantium, l'agent de la heart-water, n'a pas été mis en évidence dans les frottis de cortex cérébral.

\section{Contrôle d'efficacité}

L'un des sept bouvillons ayant reçu une dose vaccinale par injection sous-cutanée a présenté 6 jours plus tard un placard œdémateux au point d'inoculation de la largeur de la main; la lésion a régressé spontanément en quelques jours.

Un mois après la vaccination, les sérums des vaccinés possédaient des anticorps antipestiques neutralisants (titre non précisé) et une légère montée d'anticorps péripneumoniques fixant le complément et hémagglutinant les hématies sensibilisées par le polyoside charbonneux.

A l'épreuve virulente, les bovins vaccinés ont développé au point d'inoculation de la souche pathogène de petits odèmes de 3 à $7 \mathrm{~cm}$ de diamètre régressant d'eux-mêmes en l'espace de quelques jours. Par contre, les 3 témoins ont présenté un volumineux œdème de la partie latérale de l'encolure qui a nécessité leur sacrifice: ils ne pouvaient plus ni boire ni s'alimenter.

Cette fois encore, 4 sur 6 des moutons vaccinés, récemment achetés pour le test, sont morts dans un délai de 10 à 14 jours après la vaccination mais les 3 moutons témoins venant du test d'innocuité et qui, de ce fait, séjournaient en stabulation depuis plusieurs semaines, n'ont accusé aucun trouble. Après l'épreuve virulente réalisée le $15^{\mathrm{e}}$ jour suivant la vaccination, ces 3 moutons résistent parfaitement mais les 2 survivants vaccinés meurent le $2^{\mathrm{e}}$ et le $3^{e}$ jour. $B$. anthracis est réisolé mais les circonstances n'ont pas permis d'élucider s'il s'agissait de la souche vaccinale ou de la souche d'épreuve.

\section{Comportement du vaccin en solution molaire de sulfate de magnésium}

Trois essais menés comparativement sur des flacons de vaccin lyophilisé dilués soit en sérum physiologique glacé soit en solution molaire de sulfate de magnésium ont donné les mêmes résultats.

Le titre du composant pestique est le même quelque soit le diluant. Le titre du composant péripneumonique est plus élevé de $1 \log _{10}$ en solution sulfatomagnésienne, observation classique. 
Par contre, on assiste dans cette solution à une chute importante du nombre des spores, de $7.10^{6}$ à $2.10^{5}$ par dose vaccinale.

\section{Essai sur le terrain}

Les veaux vaccinés, même les plus jeunes, n'ont présenté aucun trouble morbide après la vaccination. Il n'y a eu aucune réaction locale et, chez les plus jeunes, aucune arthrite exsudative. Incidemment, ce résultat permet de conclure à l'innocuité de la souche $T_{1}-S R$ de $M$. mycoides dans cette population, chez les veaux en particulier, ainsi qu'à celle de l'association vaccinale.

Les résultats sérologiques détaillés figurent dans une autre publication (32). Deux cent trente quatre veaux avaient été vaccinés après la seconde visite, on n'a pas pu disposer de tous les sérums pour les trois réactions, soit que les propriétaires aient été partis en transhumance, soit que les tubes se soient renversés pendant leur transport au laboratoire, soit que la quantité recueillie ait été insuffisante.

a) Immunogénèse bovipestique. - Sur 234 veaux disponibles lors de la vaccination, 35 possèdent des anticorps bovipestiques neutralisants : 19 âgés de plus de 12 mois, 16 âgés de 8 mois dont 14 avec des titres élevés qui indiquent, sauf pour 2, que ce ne peut être des anticorps d'origine colostrale. Dans le groupe, on a donc 35 animaux qui présentent des anticorps pestiques sans trace auriculaire de vaccination. Aucun des plus jeunes, âgés de 6 mois, n'a d'anticorps d'origine colostrale. Les animaux possédant des anticorps avant la vaccination ont été exclus du calcul pour l'appréciation de l'immunogénèse.

Sur les 147 paires de sérums sans anticorps antipestiques anti-vaccinaux dont on dispose, on assiste à une conversion sérologique pour 139; huit animaux ne répondent pas à la vaccination, sans qu'on puisse trouver d'explication logique à ce défaut dans l'immunogénèse; on est conduit à penser que pour eux l'injection, pratiquée dans les conditions du travail en brousse, a été mal faite; extrêmement rares, en effet, sont les bovins qui n'ont pas de conversion sérologique après inoculation d'un vaccin antibovipestique.

La qualité des anticorps est bonne. Le $\mathrm{TN}_{50}$ est de $2,47 \pm 0,12$ (intervalle de confiance donné à 95 p. 100). b) Immunogénèse péripneumonique. - On dispose de 180 paires de sérums.

Cent soixante dix animaux ont avant vaccination des anticorps fixant le complément (technique de Farcha) à faible titre; cette situation est normale avec le type de réaction sérologique, de très grande sensibilité.

On constate que la conversion sérologique est fort inconstante et de faible intensité; elle ne touche que 59 animaux et n'intervient que pour 1 ou 2 dilutions des sérums. En revanche, 26 sérums voient leur titre baisser d'une dilution' de la première à la $2^{\mathrm{e}}$ saignée.

La moyenne de la différence des titres (exprimés en $\log _{2}$ ) avant et après vaccination est de $0,39 \pm 0,19$, donc intrinsèquement très faible. L'inconstance et la faiblesse de la conversion sérologique est un fait bien établi avec la souche $T_{1}$-SR (4); elle ne doit pas surprendre mais, surtout, on ne doit en tirer aucune conclusion défavorable quant à l'immunité subséquente.

c) Immunogénèse charbonneuse. - On constate qu'avant la vaccination 75 sérums sur les 136 paires examinées possèdent des anticorps actifs en hémagglutination indirecte. Il paraît difficile de trouver une explication à leur origine: manque de spécificité de l'antigène mis en cuvre ou de l'anticorps détecté, contact antérieur avec l'antigène charbonneux ? Une vaccination anticharbonneuse méconnue paraît pourtant pouvoir être exclue car, devant avoir été faite par une équipe volante de vaccination qui aurait nécessairement vacciné en même temps contre la peste, on devrait trouver ces anticorps charbonneux uniquement chez les veaux à sérologie pestique positive. Non seulement ce n'est pas le cas, mais la moitié des veaux à sérologie pestique positive, que l'on suppose avoir été vaccinés, n'hébergent pas d'anticorps charbonneux lors de la première prise de sang.

D'une manière générale, la vaccination avec le vaccin mixte est suivie soit d'une conversion sérologique soit d'une montée importante d'anticorps. La moyenne de la différence des titres (exprimés en $\log _{2}$ ) avant et après vaccination est de 1,64 $\pm 0,57$.

Pourtant, on remarque que 25 sérums ont le même titre avant qu'après la vaccination (12 sans anticorps, 13 avec anticorps à des titres variables) et que 28 accusent une baisse de titre lors de la $2^{\text {e }}$ saignée. Comme ce ne 
sont pas spécialement les animaux qui n'ont pas répondu à l'antigène bovipestique qui voient leur sérologie étalée ou diminuée, il est difficile d'accuser la vaccination en elle-même. Par ailleurs, cette catégorie d'animaux est répartie au hasard dans les groupes d'animaux vaccinés; on ne peut dès lors non plus penser à l'action néfaste pour les spores charbonneuses des antibiotiques résiduels dans le vaccin reconstitué prêt à l'emploi; si tel était le cas, on aurait une conversion sérologique anticharbonneuse des animaux vaccinés les premiers dans la séance de vaccination et aucune montée d'anticorps pour les derniers; explication qui, au demeurant, ne serait pas valable pour ceux qui ont une diminution de leurs anticorps.

On est alors conduit à incriminer la spécificité de la réaction d'hémagglutination indirecte utilisée, réaction qui avait été mise au point spécialement pour cette expérience mais pour laquelle, hormis cet essai, on n'a pas d'éléments de jugement. On conçoit combien il aurait été intéressant de pouvoir faire des épreuves d'immunité.

\section{DISCUSSION ET CONCLUSION}

Le vaccin mixte trivalent antibovipestique antipéripneumonique, anticharbonneux proposé avait une motivation bien précise qui était la vulgarisation à grande échelle de la vaccination anticharbonneuse au Tchad pour diminuer l'incidence de l'enzootie.

La solution proposée est simple et ne requiert pas une technologie poussée puisque la seule précaution à prendre est la réfrigération des constituants du vaccin lors de la préparation du mélange des antigènes. A un moment, on a songé à l'adjonction de pénicillinase, enzyme obtenue industriellement à partir de jus de culture de Bacillus cereus pour un prix modique. Toutefois, la pénicillinase n'est active qu'entre 25 et $40^{\circ} \mathrm{C}$; il aurait donc fallu travailler avec un mélange vaccinal maintenu à la température du laboratoire et on courait alors le risque de l'altération de l'antigène charbonneux par la streptomycine résiduelle lors du début de germination des spores dans le vaccin. On s'est en définitive arrêté à la méthodologie décrite.

Les contrôles des titres des différents constituants dans le vaccin reconstitué en sérum physiologique glacé sont satisfaisants et sont conformes aux normes internationales. A ce sujet, il paraît utile de préciser que les déboires connus par certains expérimentateurs $(13,22)$ dans la lyophilisation des vaccins antipéripneumoniques tiennent plus à de la technologie qu'à de véritables difficultés scientifiques. Le succès paraît résider dans la lyophilisation primaire à basse température $\left(-25^{\circ} \mathrm{C}\right)$, ce qui n'est possible qu'avec certains types de lyophilisateurs maintenant cette température sur les étagères supportant les flacons de vaccin. Les dizaines de millions de doses de vaccin antipéripneumonique ou mixte antipestique-antipéripneumonique déjà produites par certains laboratoires africains et utilisées avec succès sur le terrain sont là pour affirmer le bien fondé de cette position.

Le vaccin mixte se révèle inoffensif pour les bovins. Il n'en est apparemment pas de même pour le mouton, ce qui au demeurant est sans importance pratique puisque le vaccin trivalent n'a pas d'indication pour cette espèce. L'observation de la mortalité différée des moutons après vaccination est pourtant intrigante. $\mathbf{L a}$ souche Sterne de $B$. anthracis ne paraît pas être en cause, car des tests d'innocuité et d'efficacité se font couramment chez cette espèce; de rares rapports (Rajagopalan et Israil, 1952) font état d'accidents. Par contre, il est d'observation courante que les petits ruminants supportent mal la claustration imposée au laboratoire et qu'une importante mortalité non spécifique suit la mise en étable; tel était le cas lorsque, au laboratoire, on préparait un vaccin antirabique formolé à partir d'encéphales de mouton. Tel paraît bien être le cas dans les tests relatés plus haut, où les moutons sont morts dans les jours suivant la vaccination alors que trois de leurs congénères, habitués à l'étable, la supportent parfaitement et résistent à l'épreuve. Quelle que soit la cause, la conclusion pratique est que les tests d'efficacité ne peuvent se faire sur mouton. L'espèce bovine étant relativement insensible au charbon bactéridien expérimental, c'est au cobaye que l'on songe, mais cette possibilité n'a pas encore été explorée.

Le vaccin trivalent avait été conçu pour le bénéfice du Service de l'Elevage du Tchad, service qui dans la pratique courante utilise la solution molaire de sulfate de magnésium pour la reconstitution des vaccins antipestique et mixte antipestique-antipéripneumonique sur le terrain, pour les raisons que l'on a déjà dites. 
Les contrôles effectués indiquent amplement que l'antigène charbonneux du vaccin mixte est détérioré par la solution sulfatomagnésienne. En conséquence, le vaccin mixte décrit ne trouve pas son indication au Tchad, conclusion décevante pour le chercheur. Il n'en reste pas moins que le vaccin trivalent conserve son indication pour les Etats qui continuent à utiliser la réfrigération en glace fondante pour le transport des vaccins lyophilisés sur le terrain et surtout le rafraîchissement des diluants de reconstitution des vaccins lyophilisés. De nombreux Etats d'Afrique occidentale sont dans ce cas.

L'immunogénèse vaccinale paraît être correcte après vaccination. La présente étude n'a pas envisagé d'épreuves bovipestique et péripneumonique car on savait amplement ce que l'on était en droit d'attendre de la vaccination avec ces deux antigènes pourvu que le titre requis dans la dose vaccinale soit atteint $(31,6)$. L'épreuve vaccinale effectuée sur bovins pour l'immunogénèse charbonneuse indique que l'on peut compter sur une protection valable.

On sera plus circonspect pour l'interprétation des résultats sérologiques obtenus avec la réaction d'hémagglutination indirecte pour la recherche des anticorps charbonneux. PALIT et NILAKANTAN (21), utilisant un test sérologique similaire, observent eux aussi de nombreux sérums " normaux " possédant des anticorps anticharbonneux. Il est vraisemblable que l'on se trouve devant une réactivité sérologique de groupe; des constituants polyosidiques soma- tiques (la souche Sterne utilisée pour la préparation de l'antigène n'étant pas capsulée) aux propriétés antigéniques voisines existent en effet chez $B$. anthracis et de nombreux bacilles anthracoïdes, dont Bacillus cereus (11).

D'autre part, on n'observe d'augmentation du titre des anticorps que pour 61 p. 100 des sérums des veaux vaccinés, alors que près de 15 p. 100 ont leur titre égal et que 24 p. 100 le voient diminuer. Il paraît imprudent de vouloir tabler sur ces résultats disparates tant que l'on ne sait pas plus ce que l'on est en droit d'attendre de l'hémagglutination indirecte dans l'appréciation de l'immunité charbonneuse. L'antigène protéique de BUCHANAN (1) aurait peut-être apporté des résultats plus convaincants par la plus grande spécificité que l'on est en droit d'en espérer. Dans l'immédiat, il est préférable d'utiliser la classique épreuve d'immunité pour contrôler le vaccin.

Au total, le vaccin mixte trivalent proposé, s'il ne remplit pas totalement le but que l'on voulait atteindre au $\mathrm{T}$ chad, se révèle tout de même un immunogène valable et commode pour les services vétérinaires qui utilisent la glace hydrique sur le terrain. Pour le Tchad, il faudra songer à d'autres " cocktails " vaccinogènes; l'un est le vaccin antipestique-antipéripneumonique déjà largement utilisé; l'autre pourrait être une association charbon symptomatique-charbon bactéridien actuellement à l'étude. On doit garder la foi dans les associations vaccinales, tellement elles se révèlent précieuses pour l'utilisateur et prometteuses pour le cheptel.

\section{SUMMARY}

\section{Trivalent vaccine against rinderpest, contagious bovine pleuropneumonia and anthrax}

The authors give the reasons of the use of a combined rinderpestpleuropneumonia-anthrax vaccine in Central Africa, then they describe the idea for the combination and the methodology for its preparation. Production controls and field trials are commented upon. Molar magnesium sulphate which is used in Chad for reconstitution of the combined rinderpest-pleuropneumonia vaccine is injurious to the $B$. anthracis spores.

\section{RESUMEN}

Una vacuna mixta trivalente contre la peste bovina, la perineumonia y el carbunco bacteriano

Los autores indican las razones favorables al uso de una vacuna mixta antibovipestica, antiperineumonia y contra el carbunco en Africa Central. 
Pués describen la concepción de tal vacuna y la metodologia de su preparación.

Se comentan las pruebas de producción y los ensayos sobre terreno: la solución molar de sulfato de magnesio, utilizada en Chad para la colocación en solución de la vacuna mixta antipestica-antiperineumonia en este pais es nociva para las esporas de $B$. anthracis.

\section{BIBLIOGRAPHIE}

1. BUCHANAN (T. M.), FEELEY (J. C.), HAYES (P. S.) et BRACHMAN (P.S.). Anthrax indirect microhemagglutination test. J. Immunol., 1971, 107 : 1631-1636.

2. BUTTERY (S. H.) et PLACKETT (P.). A specific polysaccharide from Mycoplasma mycoides. J. gen. Microbiol., 1960, 23: 357-368.

3. $\mathrm{CHO}$ (H. J.). Demonstration of complement fixing antibody in the sera of cattle vaccinated with combined living blackleg-anthrax vaccine. Canad. J. comp. Med., 1971, 35: 155-160.

4. CHAMBRON (J.) et DOUTRE (M. P.). Résultats des travaux récents conduits à Dakar dans le but d'étudier selon la méthode australienne d'épreuve la valeur de l'immunité conférée par divers vaccins antipéripneumoniques utilisant les souches $\mathrm{KH}_{3} \mathrm{~J}, \mathbf{T}_{1}$ et $\mathrm{T}_{1}-\mathrm{SR}$ (streptomycino-résistante) actuellement utilisées en Afrique de l'Ouest. Bull. epiz. Dis. Afr., 1972 (numéro spécial sur la péripneumonie) :47-55.

5. DALEEL (E. E.) et LINDLEY (E. P.). Contagious bovine pleuropneumonia: a comparison of three culture vaccines. Sud. J. vet. Sci. anim. Husb. 1970, 11: 34-40.

6. DOUTRE (M.P.), CHAMBRON (J.) et BOURDIN (P.). Valeur de l'immunité conférée par un vaccin mixte antibovipestique-antipéripneumonique lyophilisé préparé à l'aide de la souche T.-SR. Rev. Elev. Méd. vét. Pays trop., 1972, 25 : 1-14.

7. FINE (J.), EYQUEM (A.) et MAILLOUX (M.). Les hétéro-agglutinines dans le règne animal. Ann. Inst. Past., 1954, 87 : 74-83.

8. GAMBLES (R.M.). Studies on contagious bovine pleuropneumonia with special reference to the complement fixation test. Brit. vet. J., 1956, 112 : 34-40.

9. GLADSTONE (G. P.). Immunity to anthrax : Production of cell free protective antigen in cellophane sacs. Brit. J. exp. Path., 1948, 29 : 379.

10. GILBERT (F. R.), WINDSOR (R.S.). The immunizing dose of $T_{1}$ strain Mycoplasma mycoides against contagious bovine pleuropneumonia. Trop. anim. Hlth. Prod., 1971, 3 : 71-76.

11. IVANOVICS (G.) et FOLDES (J.). An immunospecific substance of Bacillus cereus similar to polysaccharide obtained from Bacillus anthracis. Naturwissenschaften, 1958, $45: 15$.

12. KARST (O.). A comparison of 2 vaccines against contagious bovine pleuropneumonia. Res. vet. Sci., 1971, $12: 18-22$.

13. KARST (O.). Contagious bovine pleuropneumonia vaccine : lyophilised $\mathbf{T}_{1}$ vaccine. Bull. epiz. Dis. Afr., 1972 (numéro spécial sur la péripneumonie) 69-76.

14. LEPISSIER (H. E.). Rapport technique général sur la campagne conjointe O.U.A./C.S.T.R. contre la peste bovine en Afrique centrale et de l'Ouest (1961-1969). Lagos, O.U.A./S.T.R.C., 1971 (Publication $\mathrm{n}^{\circ}$ 103).
15. LINCOLN (R. E.), WALKER (J. R.), KLEIN (F.) et HAINES (F. K.). Anthrax. Adv, vet. Sci., $1964,9: 327-368$.

16. LINDLEY (E. P.). Simultaneous vaccination of cattle with contagious bovine pleuropneumonia and goat-adapted rinderpest vaccine. Bull. epiz. Dis. Afr., 1967, 15 : 221-226.

17. LINDLEY (E. P.). La spiramycine et les lésions postvaccinales au vaccin lyophilisé \& $M$. $m y$ coides var. mycoides, souche $\mathrm{T}_{1} / 44^{\prime \prime}$ contre la péripneumonie contagieuse des bovidés. $C a h$. Méd. vét., 1971, 40 : 233-236.

18. MORNET (P.), ORUE (J.) et DLAGNE (L.). Persistance in vivo, dans le tissu conjonctif souscutané, du virus péripneumonique et vaccinations différées, avec des vaccins vivants. Bull. Acad. vét. Fr., 1947, 20 : 467-471.

19. Normes relatives au vaccin anticharbonneux sporulé (vivant, à usage vétérinaire). (19e rapport des experts de la standardisation biologique). Genève, O.M.S., 1967. (Rapport technique O.M.S. $n^{\circ} 361$ ).

20. Normes relatives au vaccin anti-peste bovine (vivant) préparé en cultures cellulaires et au vaccin anti-peste bovine (vivant) préparé sur l'animal. (22e rapport des experts de la standardisation biologique). Genève, O.M.S., 1970. (Rapport technique O.M.S. $\pi^{\circ}$ 444).

21. PALIT (A.) et NILAKANTAN (P. R.). Antibody response in animals immunized with different types of anthrax vaccine. Ind. J. vet. Sci., 1968, 38: 431-442.

22. PEARSON (C.W.) et LLOYD (L. C.). Freezedrying of the $\mathrm{KH}_{3} \mathrm{~J}$ strain of Mycoplasma mycoides. Bull. epiz. Dis. Afr., 1971, 19: 117-122.

23. PLOWRIGHT (W.) et FERRIS (R. D.). Studies with rinderpest virus in tissue culture. II. Pathogenicity for cattle of culture-passaged virus. J. comp. Path., 1959, 69: 173-184.

24. PLOWRIGHT (W.) et FERRIS (R. D.). Studies with rinderpest virus in tissue culture. III. The stability of cultured virus and its use in virus neutralisation tests. Arch. ges. Virus f, 1961, 11: $516-533$.

25. PLOWRIGHT (W.), HERNIMAN (K. A. J.) et RAMPTON (C.S.). Studies on rinderpest culture vaccine. II. Factors influencing the accuracy of vaccine potency tests. Res. vet. Sci., 1969, 10 : 502-508.

26. PRIESTLEY (F. W.). Report to the government of the Sudan on contagious bovine pleuropneumonia. Rome, F.A.O., 1958. (F.A.O. report $n^{0} 854$ ).

27. PROVOST (A.). Rapport annuel de la Région de recherches vétérinaires de l'Afrique centrale (Laboratoire de Farcha), 1965, p. 62.

28. PROVOST (A.). Principes de production d'un vaccin mixte associé antibovipestique-antipéripneumonique inoculé en un seul temps. Bull. epiz. Dis, Afr., 1969, $17: 7-10$.

29. PROVOST (A.). Activité thermoprotectrice de la solution molaire de sulfate de magnésium sur 
l'inactivation thermique de Mycoplasma mycoides en phase liquide. C.R. Acad. Sci., Paris, 1970, 270 D : 3156-3157.

30. PROVOST (A.) et BORREDON (C.). Un yaccin mixte antibovipestique-antipéripneumonique lyophilisé utilisable sur le terrain sans réfrigération. I. Sélection de virions bovipestiques à inactivation thermique retardée. Rev. Elev. Méd. vét. Pays trop., 1972, 25 : 507-520.

31. PROVOST (A.), BORREDON (C.) et QUEVAL (R.). Recherches immunologiques sur la péripneumonie. XI. Un vaccin mixte antıbovipestique-antipéripneumonique inoculé en un seul temps. Bull. Off. int. Epiz., 1969, 72 a : 165-203.

32. PROVOST (A.), TACHER (G.), BORREDON (C.). Recherche de l'activité immunostimulante de trois dérivés à action anthelminthique de l'Imidazole sur les immunogénèses bovipestique, péripneumonique et charbonneuse. Rev. Elev. Méd. vét. Pays trop., 1974, 27 (1) : 39-52.

33. QUEVAL (R.), PROVOST (A.) et VILLEMOT (J. M.). Comparaison de méthodes de déviation du complément utilisées dans l'étude de la péripneumonie bovine. Bull. epiz. Dis. Afr., 1964, 12: 159-170.

34. ROBIN (P.) et BOURDIN (P.). Note sur l'action du sulfate de sodium, du sulfate de magnésium et du chlorure de magnésium sur le virus de la peste bovine adapté aux cultures cellulaires. Rev. Elev. Méd. vét. Pays trop., 1966, 19: 451456.

35. SIROL (.J), DELPY (P.) et GUARD (O.). Une épidémie de charbon humain. A propos de 25 cas observés à l'hôpital de Fort-Lamy, Tchad. Presse méd., 1971, 79: 1635-1638.

36. STERNE (M.). The use of anthrax vaccine prepared from avirulent (uncapsulated) variants of Bacillus anthracis. Onderstepoort J. vet. Sci., 1939, 13: 307-312.

37. STERNE (M.). Anthrax. In: STABLEFORTH (A.W.) et GALLOWAY (I. A.). Infectious diseases of animals. Diseases due to Bacteria. London, Butterworths Scientific Publications, 1959.

38. VIDEAU (D.). Pénétration, site et mode d'action des antibiotiques chez la bactérie. Cah. Méd. vét., 1968, 37: 3-18. 